\title{
Programmed cell death factor 4 enhances the chemosensitivity of colorectal cancer cells to Taxol
}

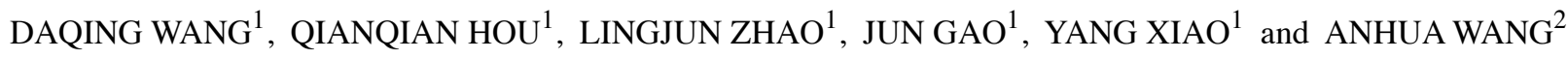 \\ ${ }^{1}$ Department of Medical Oncology, Harleeson International Peace Hospital, Hengshui, Hebei 053000; \\ ${ }^{2}$ Department of Gastrointestinal Surgery, Sanmen County People's Hospital, Taizhou, Zhejiang 317100, P.R. China
}

Received December 27, 2017; Accepted August 13, 2018

DOI: $10.3892 / \mathrm{ol} .2019 .10398$

\begin{abstract}
Drug resistance and disease relapse are still challenging problems in the chemotherapy of colorectal cancer (CRC). Programmed cell death factor 4 (PDCD4) has previously been reported to act as a tumor suppressor and was implicated in the chemosensitivity of numerous types of human malignancy. In this study, the effect of PDCD4 in the sensitivity of CRC to the chemotherapy drug Taxol was investigated. The results confirmed that lower PDCD4 expression was present in CRC tumor tissues, when compared with in normal adjacent tissues (p) and closely associated with the prognosis of patients with CRC. Upregulation of PDCD4 significantly enhanced the sensitivity of CRC cells to Taxol, by partially contributing to pro-apoptosis and anti-invasion pathways, both through upregulation of the apoptosis-associated protein Bax, and downregulation of the anti-apoptosis protein Bcl-2 and invasion-associated proteins MMP-9. These findings might present a novel strategy for sensitizing tumor cells to apoptosis and, thus, overcoming chemotherapy resistance in CRC.
\end{abstract}

\section{Introduction}

Colorectal cancer (CRC) is one of the most commonly diagnosed malignant tumors and a major cause of cancer-associated mortality worldwide (1). With its incidence increasing year by year, approximately 1.7 million new cases of CRC are estimated to be diagnosed annually by 2020 (1). Chemotherapeutics combined with surgical resection have been widely used for the treatment of CRC, and are regarded as an essential therapy for advanced-stage CRC (2). However, the therapeutic outcomes are still not optimistic. Certain patients respond poorly to the chemotherapy and continue

Correspondence to: Dr Anhua Wang, Department of Gastrointestinal Surgery, Sanmen County People's Hospital, 171 Renmin Road, Taizhou, Zhejiang 317100, P.R. China

E-mail: dr_anhuawang@163.com

Key words: colorectal cancer, programmed cell death factor 4, chemosensitivity, Taxol, apoptosis, invasion to show high rates of recurrence and distant metastasis (3). Chemoresistance is associated with the poor prognosis and is currently a major clinical problem $(4,5)$. Therefore, exploration of molecular signaling factors, including proteins or biomarkers responsible for resistance in CRC chemotherapy is crucial for the development of novel therapeutic strategies aimed at increasing the chemosensitivity of cancer cells and the patient survival rate.

Programmed cell-death factor 4 (PDCD4) is an apoptosis-associated gene (6) and is downregulated in many malignant tumors, including CRC (7-12). Its loss or downregulated expression has been found to promote tumor cell proliferation, invasion and metastasis, and to reduce tumor cell apoptosis in multiple cancer types, including cervical cancer (7), non-small cell lung cancer (NSCLC) (8), breast cancer (9), CRC (11-15), gastric cancer $(10,16)$, and esophageal squamous cell carcinoma (17), among others. Furthermore, loss or reduced PDCD4 expression has been reported to be associated with tumor progression and poor prognosis in $\operatorname{CRC}(11,12)$, NSCLC $(8,18)$ and other malignant cancers. In addition, a number of studies have shown that PDCD4 overexpression can markedly enhance the chemosensitivity of certain cancer cells, including acute myeloid leukemia (19), breast cancer (20), ovarian cancer (21), NSCLC (22), rectal cancer (23) and pancreatic cancer (24), to chemotherapy drugs. As an important tumor suppressor, multiple signaling pathways have been implicated in PDCD4-mediated cellular functions. MicroRNA-21 has been identified as the primary direct upstream regulator of PDCD4 in breast cancer (20), ovarian cancer (21), acute myeloid leukemia (19) and CRC (25). PDCD 4 has been shown to inhibit NF- $\kappa$ B signaling in order to reduce $\mathrm{NF}-\kappa \mathrm{B}$-dependent matrix metallopeptidase (MMP-9) expression in cancer cells, which can facilitate tumor cell migration and apoptosis $(26,27)$.

Despite some studies having been conducted on the effect of PDCD4 on CRC growth, invasion and metastasis, the potential modulation of PDCD4 on the chemosensitivity of CRC has not yet been reported, to the best of our knowledge. Therefore, in the present study, the effect of PDCD4 on the chemosensitivity of CRC cells to Taxol was investigated, along with the possible underlying mechanisms; this may represent a novel therapeutic target in the treatment of CRC. 


\section{Materials and methods}

Patient samples. A total of 30 matched pairs of tumor tissues and adjacent non-tumor tissues were obtained from patients with CRC who underwent surgical resection from February 2015 to June 2016 in Sanmen County People's Hospital. The study protocols were performed with approval from the Local Ethics Committee of Sanmen County People's Hospital, and with written informed consent from all patients. The clinical samples were frozen immediately in liquid nitrogen and stored at $-80^{\circ} \mathrm{C}$ until use. Details on the patients' characteristics, including age, sex, histological grade staging and metastasis status, are presented in Table I.

Cell culture. HT-29 CRC cells were obtained from the Cell Bank of Shanghai Institute of Biochemistry and Cell Biology (Chinese Academy of Sciences, Shanghai, China). Cells were grown in RPMI-1640 medium supplemented with $10 \%$ fetal bovine serum and $1 \%$ penicillin and streptomycin (all from Invitrogen; Thermo Fisher Scientific, Inc., Waltham, MA, USA), and were incubated at $37^{\circ} \mathrm{C}$ in a humidified atmosphere of $5 \% \mathrm{CO}_{2} / 95 \%$ air. Exponential growth-phase cells were routinely passaged every two days. Upon reaching 80-90\% confluence, cells were re-seeded for subsequent experiments.

Plasmid construction and cell transfection. PDCD4 cDNA was synthesized by Sino Biological, Inc. (Beijing, China), and subcloned into the restriction sites of a p-vector to generate the plasmid p-PDCD4. The constructed plasmids were verified through sequencing analysis, and then stored at $-20^{\circ} \mathrm{C}$ until use. Using Lipofectamine ${ }^{\circledR} 2000$ reagent (Invitrogen; Thermo Fisher Scientific, Inc.), cells were transiently transfected with the recombinant plasmid PDCD4 or empty vector (control) and incubated for $48 \mathrm{~h}$ at $37^{\circ} \mathrm{C}$.

Drug preparation and cell treatment. The chemotherapy drug Taxol was purchased from Tocris Bioscience (Bristol, UK), dissolved in dimethyl sulfoxide and stored at $-20^{\circ} \mathrm{C}$ until use. Cells transfected with p-PDCD4 or the empty vectors were treated with Taxol $(100 \mathrm{~nm})$. Cells treated with the empty vectors were used as the control. Subsequent assays were conducted at $48 \mathrm{~h}$ post-treatment.

Cell viability assay. Cell viability was assessed using an MTT assay using a kit purchased from Beyotime Institute of Biotechnology, Shanghai, China. Cells of each group were seeded into a 96-well plate at a density of $5 \times 10^{4}$ cells/well. A total of $10 \mu 1$ MTT (mg/ml) was added to each well, and the wells were incubated for $4 \mathrm{~h}$ at $37^{\circ} \mathrm{C}$. The optical density (OD) of each well was measured using an ELISA microplate-reader at $490 \mathrm{~nm}$.

Apoptosis assay. Cell apoptosis was evaluated using a FITC Annexin V Apoptosis Detection kit (Lonza, Inc., Allendale, NJ, USA). Cells were washed with ice-cold PBS, and resuspended in $200 \mu \mathrm{l}$ cold Annexin V binding buffer. Samples were then incubated with $5 \mu \mathrm{l}$ Annexin V conjugated with FITC and $5 \mu \mathrm{l}$ propidium iodide (PI) for $15 \mathrm{~min}$ at room temperature, and Annexin V/PI staining was detected with flow cytometry (BD FACSCalibar; BD Biosciences, San Jose, CA, USA). Data were analyzed using FlowJo software (version 9.7; Tree Star, Inc., Ashland, OR, USA).
Real-time reverse transcription-quantitative PCR ( $R T$ - $q P C R)$. Total RNA was extracted from the CRC cells or patient samples using TRIzol ${ }^{\circledR}$ reagent (Takara, Japan) and quantified using a NanoDrop-1000 spectrophotometer (Thermo Fisher Scientific, Inc.). The quality and integrity of the total RNA were examined based on OD260/280 ratio (1.8-2.1) and 2\% denaturing agarose gel electrophoresis. Total RNA was stored at $-80^{\circ} \mathrm{C}$ for the RT-qPCR assay. mRNA was reverse transcribed into cDNA using a kit (SuperScript ${ }^{\mathrm{TM}}$ IV First-Strand Synthesis system, Invitrogen; Thermo Fisher Scientific, Inc.). All PCR reactions were performed using a $20-\mu 1$ total reaction mix: $2 \mu 1$ template cDNA, $10 \mu 12 X$ SYBR Green MasterMix (MCE, Shanghai, China) and 10 pmol each primer. Relative expression of target mRNA to GAPDH was calculated using the $2^{-\Delta \Delta \mathrm{Cq}}(28)$ method to evaluate gene expression. Primers were as follows: PDCD4 forward, 5'-AAACCCTGCAGA AAATGCTGG-3' and reverse, 5'-TGCCAACACTGGTAC TCCAC-3'; GAPDH forward, 5'-GCCGCATCTTCTTTT GCGTCGC-3' and reverse, 5'-TCCCGTTCTCAGCCTTGA CGGT-3'.

Western blot analysis. CRC cells or tissue samples were lysed in RIPA buffer (Thermo Fisher Scientific, Inc.) containing a protease inhibitor cocktail (ApexBio Technology, Houston, TX, USA) on ice for $30 \mathrm{~min}$, with vortexing every $5 \mathrm{~min}$. The protein extracts were mixed with $4 \mathrm{X}$ loading buffer and denatured in a boiling water bath for $10 \mathrm{~min}$. Total proteins were separated via $10 \%$ SDS-PAGE, and then transferred to polyvinylidene difluoride membranes. The membranes were subsequently blocked with $5 \%$ skimmed milk in PBS containing $0.5 \%$ Tween 20 for $2 \mathrm{~h}$ at room temperature. Following blocking, the membranes were probed with either anti-BCL-2 (1:1,000, ab59348), anti-BAX (1:1,000, ab32503) or anti-MMP-9 (1:1,000, ab76003) antibodies (all purchased from Abcam, Cambridge, UK), or a rabbit monoclonal anti-GAPDH antibody $\left(1: 1,000\right.$, ab8245, purchased from Abcam) at $4^{\circ} \mathrm{C}$ overnight, then incubated with Goat anti-rabbit horseradish peroxidase (HRP)-conjugated secondary antibodies (1:5,000, ab6721, Abcam) at room temperature for $2 \mathrm{~h}$. Protein bands were detected using ECL reagent (EMD Millipore, Billerica, MA, USA) and imaged with a Bio-Rad Gel Doc ${ }^{\mathrm{TM}} \mathrm{XR}+$ imaging system (Bio-Rad Laboratories, Inc., Hercules, CA, USA). The intensity of target bands was quantified using ImageJ software (NIH, Bethesda, MD, USA) and normalized against GAPDH band intensity.

Statistical analysis. All results were analyzed using SPSS v.22.0 software (SPSS, Inc., Chicago, IL, USA). Values are presented as the means \pm SD from at least three independent experiments. Comparisons between two groups were performed using an unpaired Student's t-test, and comparisons among multiple groups were evaluated by one-way analysis of variance with Turkey's post-hoc test. A value of $\mathrm{P}<0.05$ was considered to indicate statistical significance.

\section{Results}

PDCD4 is downregulated in CRC tissues. Western blotting and RT-qPCR assays were conducted to analyze the expression level of PDCD4 in CRC tissue samples. As shown in Fig. 1A 
Table I. Expression of PDCD4 in CRC tissues.

\begin{tabular}{lccc}
\hline Factors & No. & PDCD4 (mean) & P-value \\
\hline $\begin{array}{l}\text { Sex } \\
\text { Male }\end{array}$ & 19 & $0.468 \pm 0.010$ & 0.966 \\
$\quad$ Female & 11 & $0.453 \pm 0.022$ & \\
Age (years) & & & \\
$\quad<60$ & 16 & $0.464 \pm 0.039$ & 0.352 \\
$\geq 60$ & 14 & $0.448 \pm 0.085$ & \\
Histological grade & & & \\
$\quad$ Well-intermediate & 10 & $0.406 \pm 0.020$ & $0.048^{\mathrm{a}}$ \\
$\quad$ differentiation & & & \\
$\quad$ Poor differentiation & 20 & $0.491 \pm 0.028$ & \\
Metastasis & & & $0.022^{\mathrm{a}}$ \\
$\quad$ No & 23 & $0.498 \pm 0.022$ & \\
$\quad$ Yes & 7 & $0.399 \pm 0.010$ & \\
\hline
\end{tabular}

${ }^{\mathrm{a}} \mathrm{P}<0.05$. PDCS4, programmed cell death factor 4 .

and B, a significantly reduced expression level of PDCD4 was observed in the tumor tissues, compared with in adjacent non-tumor controls. The association of PDCD4 expression and clinicopathological factors was subsequently investigated, as presented in Table I. It was found that lower expression of PDCD4 was significantly associated with histological grade and metastasis $(\mathrm{P}=0.048$ and 0.022 , respectively). No significant associations were observed between PDCD4 expression and sex or age. These results confirmed a strong association between low expression of PDCD4 and CRC progression, consistent with previous studies.

Upregulation of PDCD4 enhances the sensitivity of CRC cells to Taxol. To explore whether the drug responses of CRC cells might be modulated by PDCD4, CRC cells were transfected with PDCD4 plasmid or empty vector, and then treated with or without Taxol for $48 \mathrm{~h}$. Cell viability and apoptosis assays were then performed. As Figs. 2 and 3 indicate, transfection with the PDCD4 plasmid induced a significant decrease in the proliferation, and a marked increase in the apoptosis, of CRC cells. Furthermore, Taxol-treated cells exhibited a significantly reduced level of growth and an enhanced level of apoptosis; however, overexpression of PDCD4 markedly decreased growth and increased apoptosis in Taxol-treated cells. These observations strongly indicate that PDCD4 can induce a significant decrease in tumor cell survival and an increase in tumor apoptosis, leading to the enhanced sensitivity of CRC cells to Taxol treatment.

Overexpression of PDCD4 upregulates Bax and downregulates Bcl-2 and MMP-9 expression. To investigate the possible mechanisms by which PDCD4 affects cell proliferation and apoptosis, PDCD4-transfected or -non-transfected cells were treated with or without Taxol, RT-qPCR and western blotting assays were then performed to detect expression of the apoptosis-associated genes Bax and Bcl-2, and the invasion-associated molecule MMP-9. As presented in Figs. 4 and 5,

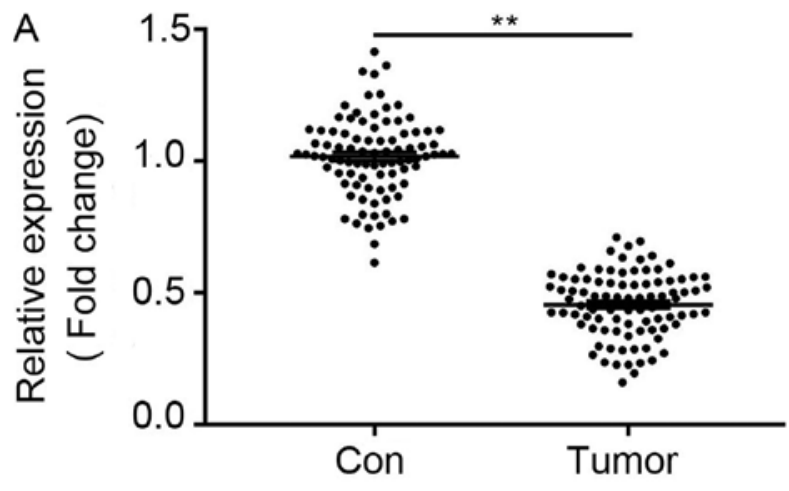

B

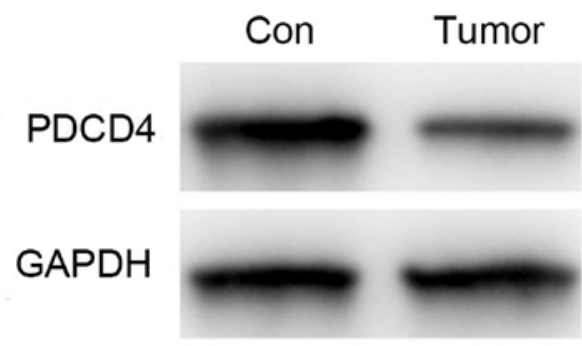

Figure 1. PDCD4 is downregulated in CRC clinical tissues. (A) Compared with in adjacent normal tissues, PDCD4 mRNA expression was significantly decreased in CRC tissues. (B) Compared with in adjacent normal tissues, PDCD4 protein expression was significantly decreased in CRC tissues. Data were normalized to the GAPDH control. Results are expressed relative to the normal control levels that were assigned a value of 1 . Data are presented as means $\pm \mathrm{SD}$. ${ }^{* *} \mathrm{P}<0.01$. PDCS4, programmed cell death factor 4 ; CRC, colorectal cancer.

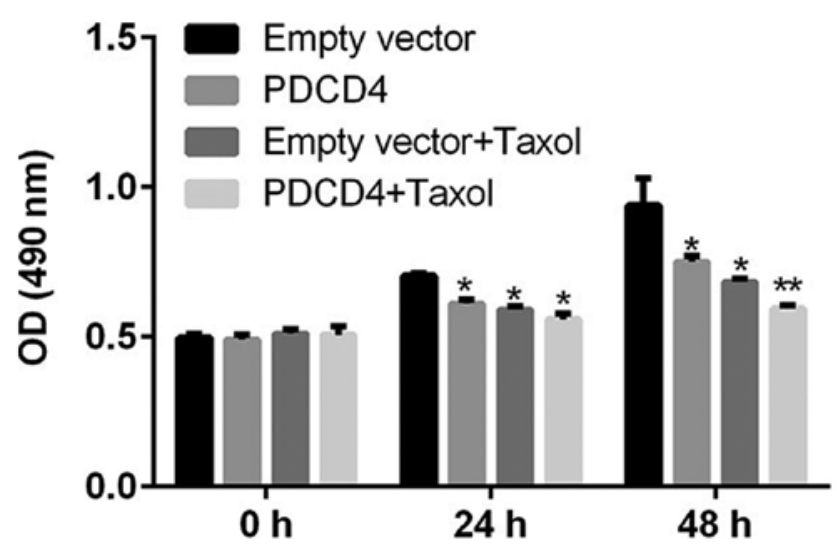

Figure 2. Effect of PDCD4 overexpression plasmid on the viability of Taxol-treated CRC cells in vitro. CRC cells were divided into four different groups: Empty vector group, PDCD4 overexpression group, Taxol treatment group, and Taxol treatment + PDCD4 overexpression group. Cell viability was examined at $48 \mathrm{~h}$ after treatment. Compared with the empty vector group, cell viability in the PDCD4 overexpression and Taxol treatment groups was significantly reduced, and further reduced by co-administration of Taxol and the PDCD4 plasmid. Data are presented as the means \pm SD of three independent experiments. ${ }^{*} \mathrm{P}<0.05,{ }^{* *} \mathrm{P}<0.01$. PDCS4, programmed cell death factor 4; CRC, colorectal cancer.

transfection with the PDCD4 plasmid lead to increased expression of Bax and decreased expression of Bcl-2 and MMP-9 on both the mRNA and protein levels. Furthermore, treatment with Taxol resulted in the upregulation of Bax and downregulation of Bcl-2 and MMP-9 protein expression levels. Notably, 

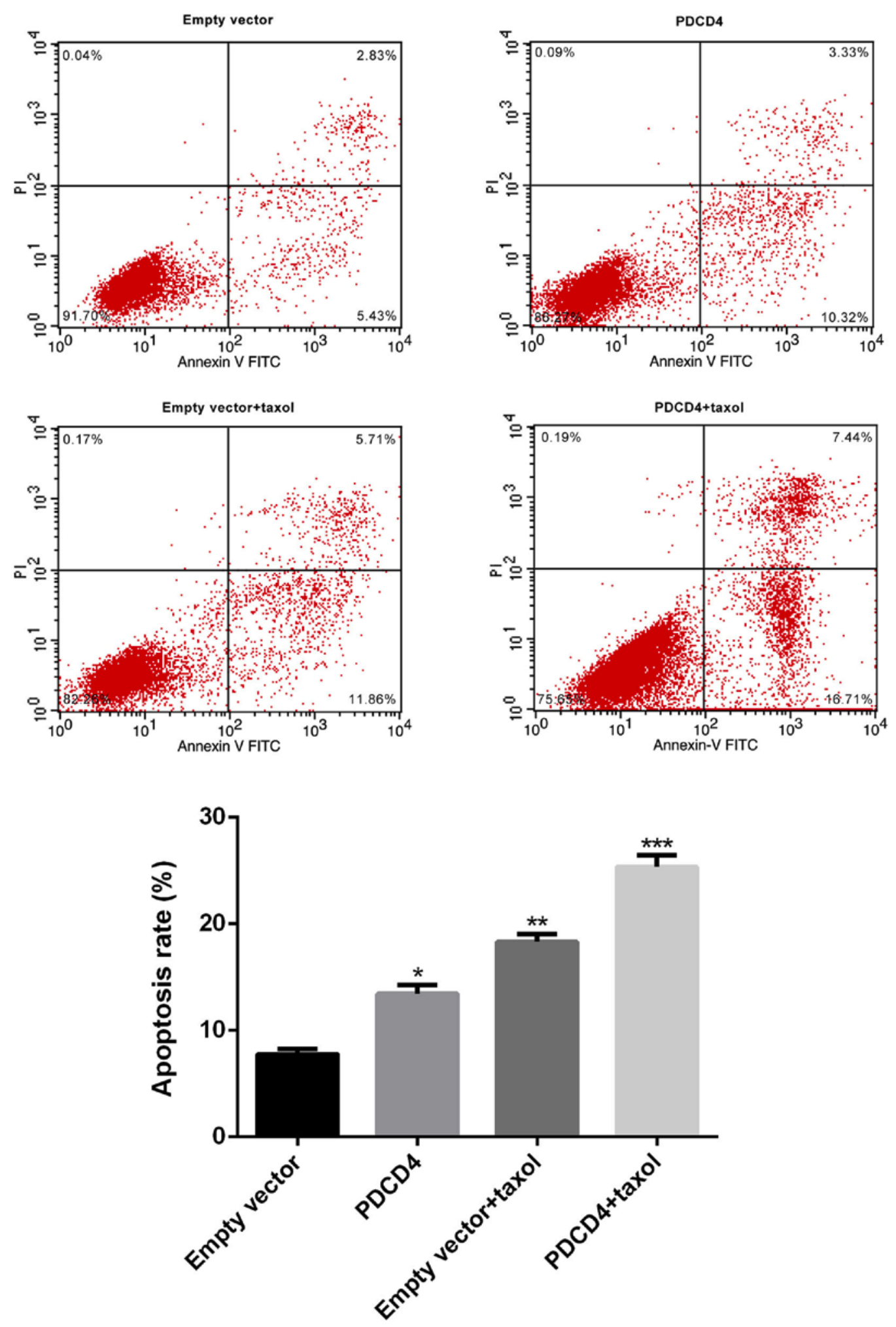

Figure 3. Effect of PDCD4 over-expression plasmid on the apoptosis of Taxol-treated CRC cells in vitro. CRC cells were divided into four different groups: Empty vector group, PDCD4 overexpression group, Taxol treatment group, and Taxol treatment + PDCD4 overexpression group. Apoptosis was analyzed at $48 \mathrm{~h}$ post-treatment by flow cytometry. Compared with the empty vector group, cell apoptosis in the PDCD4 overexpression and Taxol treatment groups was significantly increased, and further induced by co-administration of Taxol and the PDCD4 plasmid. Data are presented as the means \pm SD of three independent experiments. ${ }^{*} \mathrm{P}<0.05,{ }^{* *} \mathrm{P}<0.01,{ }^{* * *} \mathrm{P}<0.001$. PDCS4, programmed cell death factor 4; CRC, colorectal cancer.

PDCD4-transfected cells exhibited a marked increase in Bax expression and decrease in Bcl-2 and MMP-9 expression in the Taxol-treatment group. Furthermore, the ratio of Bax/Bcl-2 was notably elevated in PDCD-transfected and Taxol-treated cells compared with that in cells treated with Taxol alone. Collectively these data suggest that PDCD4 transfection and Taxol treatment can synergistically downregulate expression of Bax and upregulate the expression of Bcl-2 and MMP-9. 

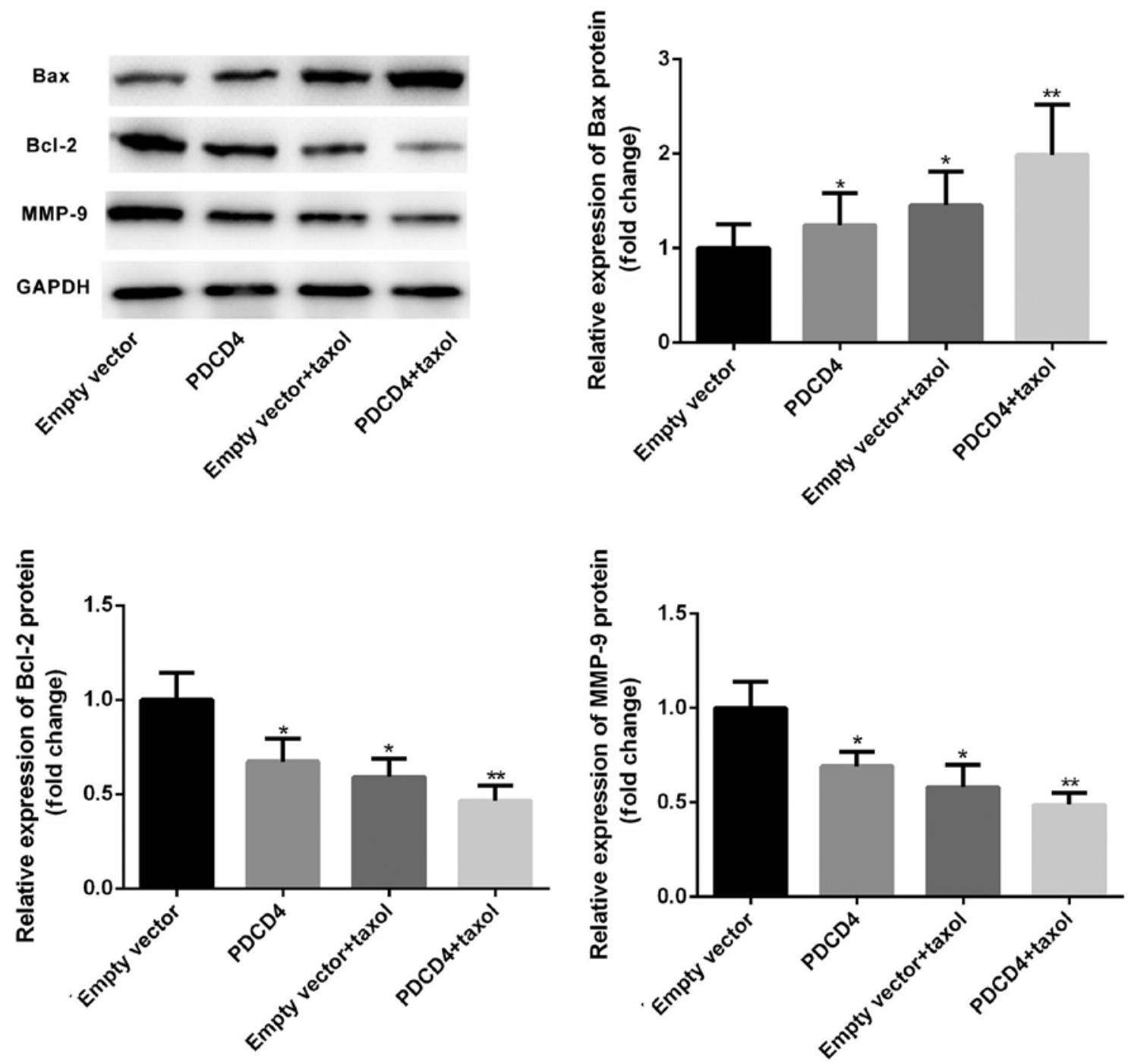

Figure 4. Relative protein expression of Bax, Bcl-2 and MMP-9. CRC cells were divided into four different groups: Empty vector group, PDCD4 overexpression group, Taxol treatment group, and Taxol treatment + PDCD4 overexpression group. At $48 \mathrm{~h}$ post-treatment, total cell lysates were assayed via western blotting with the indicated antibodies. GAPDH was used as the loading control. Compared with the empty vector group, Bax protein level in the PDCD4 overexpression and Taxol treatment groups were significantly increased, which was further induced by co-administration of Taxol and the PDCD4 plasmid. Bcl-2 and MMP-9 protein levels were significantly decreased in the PDCD4 overexpression and Taxol groups, and enhanced by co-administration of Taxol and the PDCD4 plasmid. Data are presented as the means $\pm \mathrm{SD} .{ }^{*} \mathrm{P}<0.05,{ }^{* *} \mathrm{P}<0.01$. PDCS4, programmed cell death factor 4; CRC, colorectal cancer; Bcl-2, B-cell lymphoma-2; BAX, Bcl-2-associated X protein; MMP-9, matrix metalloproteinase-9.

\section{Discussion}

At present, the treatment of CRC, especially advanced-stage $\mathrm{CRC}$, requires neoadjuvant chemotherapy following surgical resection. Taxol and 5-fluorouracil (5-FU) are the main chemotherapy drugs that are widely used in CRC therapy. With advancements in therapeutic strategies, for example, certain novel adjuvant drugs now being combined with Taxol or 5-FU, numerous patients have benefited from the chemotherapy regimens and exhibit improved overall survival. However, this therapeutic effect is limited as disease relapse can occur from drug-resistant cells. To date, chemotherapy resistance remains one of the major causes of cancer-associated mortality among patients with CRC after therapy. Therefore, it is necessary to identify effective biomarkers to target to make CRC tumor cells more sensitive to chemotherapy drugs.

PDCD4 was initially identified as an apoptosis-associated gene (29) and subsequently found to function as a tumor suppressor gene $(30,31)$. Further studies have shown that its loss or lower expression is strongly implicated in the progression and poor prognosis of various types of cancers including CRC (11,12). Multiple regulation mechanisms have been described in association with PDCD4. For example, PDCD4 was reported to interact with translation initiation factor $4 \mathrm{~A}$ (eIF4A) and eIF4G and inhibit translation (32). Furthermore, PDCD4 can be upregulated during apoptosis in response to different inducers $(29,33)$. In CRC, the regulation of PDCD4 may be negatively modulated by its upstream oncogenic molecule microRNA-21 $(11,12)$. Our data confirmed the reduced mRNA and protein expression of PDCD4 in CRC tissues, and the positive association between its low expression and the aggressiveness and development of CRC reported in previous studies (11-15).

A growing body of evidence suggests that apoptosis is closely involved in mediating cancer cell death induced by chemotherapy drugs. Upregulation of PDCD4 has been 


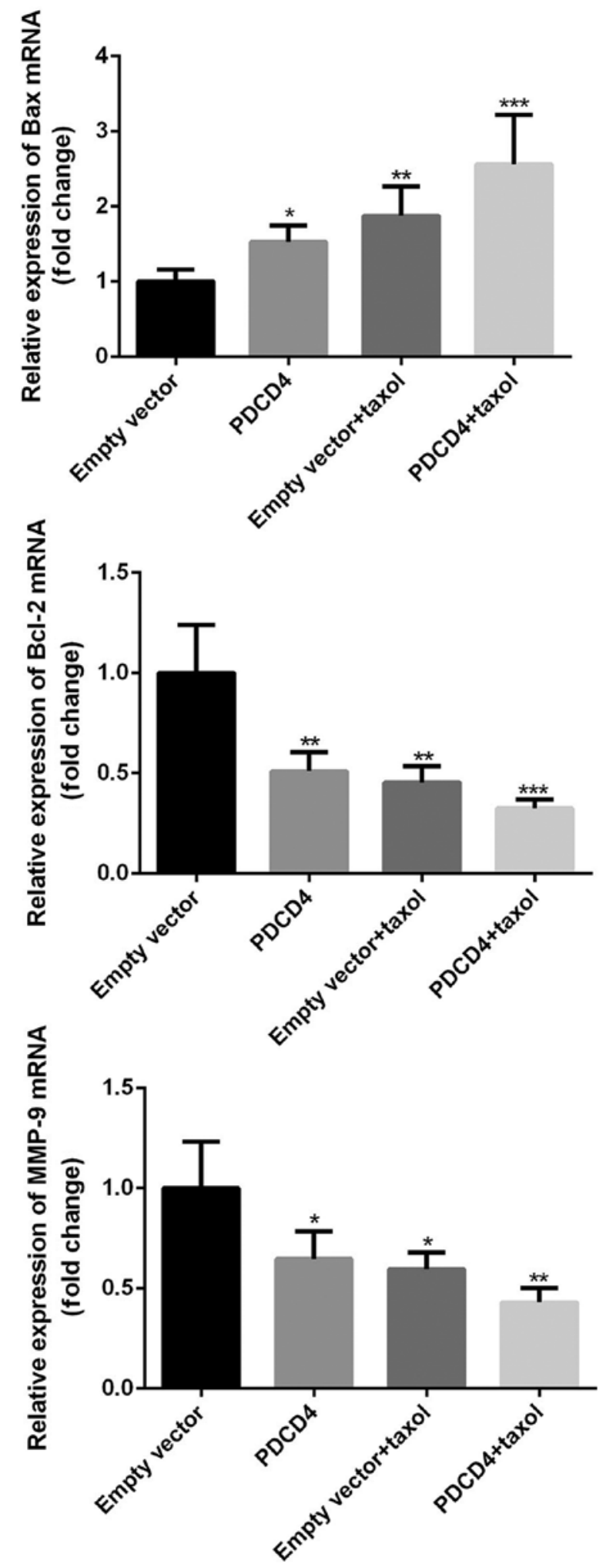

Figure 5. Relative mRNA expression of Bax, Bcl-2, and MMP-9. Relative mRNA expression of Bax, Bcl-2 and MMP-9 was analyzed using RT-qPCR. Results were normalized to GAPDH and expressed relative to the empty vector group levels that were assigned a value of 1 . Compared with the empty vector group, Bax mRNA levels in the PDCD4 overexpression and Taxol treatment groups were significantly increased, and further induced by co-administration of Taxol and the PDCD4 plasmid. Bcl-2 and MMP-9 mRNA levels were significantly decreased in the PDCD4 overexpression and Taxol groups, and enhanced by co-administration of Taxol and the PDCD4 plasmid. Data are presented as the means \pm SD of three independent experiments. ${ }^{*} \mathrm{P}<0.05,{ }^{* *} \mathrm{P}<0.01,{ }^{* * *} \mathrm{P}<0.001$. PDCS4, programmed cell death factor 4; CRC, colorectal cancer; Bcl-2, B-cell lymphoma-2; BAX, Bcl-2-associated X protein; MMP-9, matrix metalloproteinase-9. reported to elevate the sensitivity of cancer cells including NSCLC and ovarian cancer cells in response to chemotherapy drugs $(21,22)$. To date, and to the best of our knowledge, there have been no reports on the role of PDCD4 in the sensitivity of CRC cells to chemotherapy drugs. Taxol is one of the current chemotherapy drugs used for the treatment of CRC, but its use can result in chemoresistance and therapy failure (34). In the present study, PDCD4 overexpression exhibited a marked inhibitory effect on proliferation and apoptosis in CRC cells treated with Taxol at the indicated concentration. A possible mechanism underlying this effect was that PDCD4 overexpression significantly enhanced the expression of apoptosis-associated gene Bax and invasion-associated gene MMP-9, and markedly reduced the expression of the anti-apoptosis gene Bcl-2.

In the present study, lower PDCD4 expression in CRC tissues was confirmed by analysis of mRNA and protein levels, which is in line with previous reports. In addition, PDCD4 upregulation significantly elevated the sensitivity of CRC cells to the chemotherapy drug Taxol, by enhancing apoptosis and attenuating cell survival. These findings might represent a novel strategy for improving the curative effect of chemotherapy and, thus, the prognosis of patients in CRC.

\section{Acknowledgements}

Not applicable.

\section{Funding}

No funding was received.

\section{Availability of data and materials}

The datasets used and/or analyzed during the current study are available from the corresponding author on reasonable request.

\section{Authors' contributions}

DW performed the clinical and cell studies in the present study, QH and LZ also performed the clinical studies. JG performed the cell studies, and YX analyzed the data. AW designed the study and was a major contributor in writing the manuscript. All authors read and approved the final manuscript.

\section{Ethics approval and consent to participate}

The ethics approval was obtained from Sanmen County People's Hospital, and written informed consent was obtained from all patients.

\section{Patient consent for publication}

Informed consent for publication of the dataset from participants was obtained.

\section{Competing interests}

The authors declare that they have no competing interests. 


\section{References}

1. Ferlay J, Soerjomataram I, Dikshit R, Eser S, Mathers C, Rebelo M, Parkin DM, Forman D and Bray F: Cancer incidence and mortality worldwide: Sources, methods and major patterns in GLOBOCAN 2012. Int J Cancer 136: E359-E386, 2015.

2. Gustin DM and Brenner DE: Chemoprevention of colon cancer: Current status and future prospects. Cancer Metastasis Rev 21: 323-348, 2002

3. Fakih MG: Metastatic colorectal cancer: Current state and future directions. J Clin Oncol 33: 1809-1824, 2015.

4. Gieseler F, Rudolph P, Kloeppel G and Foelsch UR: Resistance mechanisms of gastrointestinal cancers: Why does conventional chemotherapy fail? Int J Colorectal Dis 18: 470-480, 2003.

5. De Rosa M, Pace U, Rega D, Costabile V, Duraturo F, Izzo P and Delrio P: Genetics, diagnosis and management of colorectal cancer (Review). Oncol Rep 34: 1087-1096, 2015.

6. Fawzy MS, Toraih EA, Ibrahiem A, Abdeldayem H, Mohamed AO and Abdel-Daim MM: Evaluation of miRNA-196a2 and apoptosis-related target genes: ANXA1, DFFA and PDCD4 expression in gastrointestinal cancer patients: A pilot study. PLoS One 12: $\mathrm{e} 0187310,2017$.

7. Zhang Z, Wang J, Li J, Wang X and Song W: MicroRNA-150 promotes cell proliferation, migration, and invasion of cervical cancer through targeting PDCD4. Biomed Pharmacother 97: 511-517, 2017.

8. Zhen Y, Li D, Li W, Yao W, Wu A, Huang J, Gu H, Huang Y, Wang $\mathrm{Y}, \mathrm{Wu} \mathrm{J}$, et al: Reduced PDCD4 expression promotes cell growth through PI3K/Akt signaling in non-small cell lung cancer. Oncol Res 23: 61-68, 2016.

9. Cuesta R and Holz MK: RSK-mediated down-regulation of PDCD4 is required for proliferation, survival, and migration in a model of triple-negative breast cancer. Oncotarget 7: 27567-27583, 2016.

10. Hu X, Wang Y, Liang H, Fan Q, Zhu R, Cui J, Zhang W, Zen K, Zhang CY, Hou D, et al: miR-23a/b promote tumor growth and suppress apoptosis by targeting PDCD4 in gastric cancer. Cell Death Dis 8: e3059, 2017.

11. Chang KH, Miller N, Kheirelseid EA, Ingoldsby H, Hennessy E, Curran CE, Curran S, Smith MJ, Regan M, McAnena OJ and Kerin MJ: MicroRNA-21 and PDCD4 expression in colorectal cancer. Eur J Surg Oncol 37: 597-603, 2011.

12. Horiuchi A, Iinuma H, Akahane T, Shimada R and Watanabe T: Prognostic significance of PDCD4 expression and association with microRNA-21 in each Dukes' stage of colorectal cancer patients. Oncol Rep 27: 1384-1392, 2012.

13. Liu Y, Uzair-Ur-Rehman1, Guo Y, Liang H, Cheng R, Yang F, Hong Y, Zhao C, Liu M, Yu M, et al: miR-181b functions as an oncomiR in colorectal cancer by targeting PDCD4. Protein Cell 7: 722-734, 2016.

14. Liu X, Zhang Z, Sun L, Chai N, Tang S, Jin J, Hu H, Nie Y, Wang X, Wu K, et al: MicroRNA-499-5p promotes cellular invasion and tumor metastasis in colorectal cancer by targeting FOXO4 and PDCD4. Carcinogenesis 32: 1798-1805, 2011.

15. Ferraro A, Kontos CK, Boni T, Bantounas I, Siakouli D, Kosmidou V, Vlassi M, Spyridakis Y, Tsipras I, Zografos G and Pintzas A: Epigenetic regulation of miR-21 in colorectal cancer: ITGB4 as a novel miR-21 target and a three-gene network (miR-21-ITGBeta4-PDCD4) as predictor of metastatic tumor potential. Epigenetics 9: 129-141, 2014.

16. Li C, Deng L, Zhi Q, Meng Q, Qian A, Sang H, Li X and Xia J: MicroRNA-183 functions as an oncogene by regulating PDCD4 in gastric cancer. Anticancer Agents Med Chem 16: 447-455, 2016.

17. Wu Y, Hu L, Liang Y, Li J, Wang K, Chen X, Meng H, Guan X Yang K and Bai Y: Up-regulation of IncRNA CASC9 promotes esophageal squamous cell carcinoma growth by negatively regulating PDCD4 expression through EZH2. Mol Cancer 16: 150, 2017.
18. Chen Y, Knösel T, Kristiansen G, Pietas A, Garber ME, Matsuhashi S, Ozaki I and Petersen I: Loss of PDCD4 expression in human lung cancer correlates with tumour progression and prognosis. J Pathol 200: 640-646, 2003.

19. Li Y, Zhu X, Gu J, Hu H, Dong D, Yao J, Lin C and Fei J: Anti-miR-21 oligonucleotide enhances chemosensitivity of leukemic HL60 cells to arabinosylcytosine by inducing apoptosis. Hematology 15: 215-221, 2010.

20. Bourguignon LY, Spevak CC, Wong G, Xia W and Gilad E: Hyaluronan-CD44 interaction with protein kinase C(epsilon) promotes oncogenic signaling by the stem cell marker nanog and the production of microRNA-21, leading to down-regulation of the tumor suppressor protein PDCD4, anti-apoptosis and chemotherapy resistance in breast tumor cells. J Biol Chem 284: 26533-26546, 2009.

21. 21.Chan JK, Blansit K, Kiet T, Sherman A, Wong G, Earle C and Bourguignon LY: The inhibition of miR-21 promotes apoptosis and chemosensitivity in ovarian cancer. Gynecol Oncol 132: 739-744, 2014.

22. Ning FL, Wang F, Li ML, Yu ZS, Hao YZ and Chen SS: MicroRNA-182 modulates chemosensitivity of human non-small cell lung cancer to cisplatin by targeting PDCD4. Diagn Pathol 9: 143,2014

23. Dou X, Wang RB, Meng XJ, Yan HJ, Jiang SM, Zhu KL, Xu XQ, Chen D, Song XR and Mu DB: PDCD4 as a predictor of sensitivity to neoadjuvant chemoradiotherapy in locally advanced rectal cancer patients. Asian Pac J Cancer Prev 15: 825-830, 2014.

24. Wei X, Wang W, Wang L, Zhang Y, Zhang X, Chen M, Wang F, Yu J, Ma Y and Sun G: MicroRNA-21 induces 5-fluorouracil resistance in human pancreatic cancer cells by regulating PTEN and PDCD4. Cancer Med 5: 693-702, 2016.

25. Asangani IA, Rasheed SA, Nikolova DA, Leupold JH, Colburn NH, Post S and Allgayer H: MicroRNA-21 (miR-21) post-transcriptionally downregulates tumor suppressor Pdcd4 and stimulates invasion, intravasation and metastasis in colorectal cancer. Oncogene 27: 2128-2136, 2008.

26. Parks WC, Wilson CL and Lopez-Boado YS: Matrix metalloproteinases as modulators of inflammation and innate immunity. Nat Rev Immunol 4: 617-629, 2004.

27. Mao XH, Chen M, Wang Y, Cui PG, Liu SB and Xu ZY: MicroRNA-21 regulates the ERK/NF-kappaB signaling pathway to affect the proliferation, migration and apoptosis of human melanoma A375 cells by targeting SPRY1, PDCD4 and PTEN. Mol Carcinog 56: 886-894, 2017.

28. Livak KJ and Schmittgen TD: Analysis of relative gene expression data using real-time quantitative PCR and the $2-\Delta \Delta \mathrm{Ct}$ method. Methods 25: 402-408, 2001.

29. Shibahara K, Asano M, Ishida Y, Aoki T, Koike T and Honjo T: Isolation of a novel mouse gene MA-3 that is induced upon programmed cell death. Gene 166: 297-301, 1995.

30. Cmarik JL, Min H, Hegamyer G, Zhan S, Kulesz-Martin M, Yoshinaga H, Matsuhashi S and Colburn NH: Differentially expressed protein Pdcd4 inhibits tumor promoter-induced neoplastic transformation. Proc Natl Acad Sci USA 96: 14037-14042, 1999.

31. Jansen AP, Camalier CE and Colburn NH: Epidermal expression of the translation inhibitor programmed cell death 4 suppresses tumorigenesis. Cancer Res 65: 6034-6041, 2005.

32. Singh P, Marikkannu R, Bitomsky N and Klempnauer KH: Disruption of the Pdcd4 tumor suppressor gene in chicken DT40 cells reveals its role in the DNA-damage response. Oncogene 28: 3758-3764, 2009.

33. Schlichter U, Burk O, Worpenberg S and Klempnauer KH: The chicken Pdcd4 gene is regulated by v-Myb. Oncogene 20: 231-239, 2001.

34. Liu Y, Gao S, Chen X, Liu M, Mao C and Fang X: Overexpression of miR-203 sensitizes paclitaxel (Taxol)-resistant colorectal cancer cells through targeting the salt-inducible kinase 2 (SIK2). Tumour Biol 37: 12231-12239, 2016. 